\title{
Time series modelling of increased soil temperature anomalies during long period
}

\author{
Amin Shirvani ${ }^{*}$, Farzad Moradi ${ }^{2}$, and Ali Akbar Moosavi ${ }^{2}$ \\ ${ }^{1}$ Department of Water Engineering, Oceanic and Atmospheric Research Centre, College of Agriculture, \\ Shiraz University, Shiraz, Iran \\ ${ }^{2}$ Department of Soil Science, College of Agriculture, Shiraz University, Shiraz, Iran
}

Received May 5, 2015; accepted October 12, 2015

\begin{abstract}
A b s t r a c t. Soil temperature just beneath the soil surface is highly dynamic and has a direct impact on plant seed germination and is probably the most distinct and recognisable factor governing emergence. Autoregressive integrated moving average as a stochastic model was developed to predict the weekly soil temperature anomalies at $10 \mathrm{~cm}$ depth, one of the most important soil parameters. The weekly soil temperature anomalies for the periods of January1986-December 2011 and January 2012-December 2013 were taken into consideration to construct and test autoregressive integrated moving average models. The proposed model autoregressive integrated moving average $(2,1,1)$ had a minimum value of Akaike information criterion and its estimated coefficients were different from zero at 5\% significance level. The prediction of the weekly soil temperature anomalies during the test period using this proposed model indicated a high correlation coefficient between the observed and predicted datathat was 0.99 for lead time 1 week. Linear trend analysis indicated that the soil temperature anomalies warmed up significantly by $1.8^{\circ} \mathrm{C}$ during the period of $1986-2011$.

$\mathrm{K}$ e y w o r d s: soil temperature anomalies time series, ARIMA model, prediction
\end{abstract}

\section{INTRODUCTION}

Temporal and spatial distribution of soil temperature relative to the growth response of both desirable plant species and weedy competitors are among the most important critical microclimate seedbed factors determining the success of seeding efforts (Roundy and Call, 1988). Microclimatic requirements for seedling establishment are much more restrictive than the conditions necessary forpersistence ofmature perennial vegetation. The system requirements for establishment of a plant community cannot be predicted without characterisation of the temporal and spatial varia-

*Corresponding author e-mail: am shirvani@hotmail.com, ashirvani@shirazu.ac.ir bility in microclimatic conditions in the field for vegetated or bare-soil conditions (Flerchinger and Hardegree, 2004). Computer simulation permits continuous monitoring of the temperature regime of the seedbed and characterisation of historical and potential future patterns of seedbed microclimate (Hardegree et al., 2003). Also, a model tested for its capability to adequately predict soil temperature could be particularly useful in order to test global warming scenario (Houle et al., 2002) and effects on some variables such as nitrogen transformation and thermal and biological degradation (Schütt et al., 2014; Subke and Bahn, 2010; Toosi et al., 2014).

Computer science and statistics have improved modelling approaches for discovering patterns found in temperature time series data. Much effort has been devoted over the past several decades to develop time series models and to improve the accuracy of their predictions. Since the selection of the 'best-fitting model' does not necessarily imply 'best forecasting performance', the choice of the forecasting technique depends on a variety of factors, such as the objective in producing forecasts, the degree of accuracy required, and the properties of the given time-series (Chatfield, 2013). One of the most important and widely used time series model is the autoregressive integrated moving average (ARIMA) model (Shahwan and Odening, 2007). This modelling approach is particularly useful when little knowledge is available on the underlying data generating process or when there is no satisfactory explanatory model that relates the prediction variable to other explanatory variables (Zhang, 2003). The popularity of the ARIMA model is due to its statistical properties as well

(C) 2015 Institute of Agrophysics, Polish Academy of Sciences 
as the well-known Box-Jenkins methodology (Box and Jenkins, 1976) in the model-building process. In addition, various exponential smoothing models can be implemented by ARIMA models (McKenzie, 1984). ARIMA models have been widely used for air quality (Diaz-Robles et al., 2008), water quality (Ömer Faruk, 2010), runoff coefficients (Pektaş and Kerem Cigizoglu, 2013), solar irradiance and surface air temperature (Kärner, 2009), soil salt and water content (Aljoumani et al., 2012; Zou et al., 2010), soil dryness index (Li et al., 2003), soil heat flux (Kumar et al., 2009) and global near-surface mean temperature (Romilly, 2005).

Soil temperature immediately beneath the soil surface is highly dynamic and has a direct impact on plant seed germination and, probably, is the most distinct and recognisable factor governing emergence (Dorado et al., 2009; Harvey and Forcella, 1993). Also, soil microorganisms are more active in the upper than in the deeper soil layers. For example, Persson and Wirén (1995) found that, on average, $78 \%$ of the net $\mathrm{N}$ mineralisation occurred above the depth of $10 \mathrm{~cm}$ and $22 \%$ occurred in the $10-50 \mathrm{~cm}$ layer in forest soil. To the best of our knowledge, no research has been done on applying the ARIMA model for studying temporal variability of soil temperature. Furthermore, the high ability of ARIMA model in recognising the patterns of temporal variations and its abovementioned accurate predictions motivated us to develop such a model for describing the temporal variability of long-ranged soil temperature data at $10 \mathrm{~cm}$ depth.

\section{MATERIAL AND METHODS}

Daily soil temperature data at $10 \mathrm{~cm}$ depth were obtained from the meteorological station at the Agricultural Research Station of Shiraz University, Bajgah, for a long time period of 28 years from 1 January 1986 to 31 December 2013. The latitude, longitude and elevation of this station which is located in the south of Iran are $29^{\circ} 44^{\prime} 55^{\prime \prime} \mathrm{N}, 52^{\circ}$ $34^{\prime} 20^{\prime \prime} \mathrm{E}$ and $1810 \mathrm{~m}$ a.s.l., respectively.

In the present paper, a model from the ARIMA family was fitted to the daily data series of soil temperature using a time step of more than one day. The longer time step leads to better fitting conditions because the soil temperature data series are, with varying intensity, non-stationary in terms of the self-similarity parameter. The latter is determined by means of the structure function. For this topic readers are referred to the detailed description by Monin et al. (1976) and Kärner (2009). Therefore, the daily time series soil temperature data were first averaged to obtain the weekly data. The average of the first and second seven days of January 1986, respectively, was considered as the first and second weekly data. This moving average with length of seven days was continued up to the last day of 1986 . This process was performed separately for each year and then the weekly time series data of soil temperature were provided. In this manner, each year contains 52 weeks. Therefore, the whole time series contains $1456=52 \times 28$ weeks. Figure 1 shows the time series plot of the weekly soil temperature (ST), indicating seasonal variations with period of 52 weeks. To eliminate seasonal variations, the weekly soil temperature anomalies were then computed with respect to weekly average for the whole studied period (1986-2013). Figure 2 shows the time series plot of the weekly soil temperature anomalies (STA) for the whole studied period. This time series was divided into two periods consisting of the first week of January 1986 to the last week of December 2011 (1352 weeks), and from the first week of January 2012 to the last week of December 2013 (104 weeks). While the first period was used for constructing the ARIMA models, the later one was applied for model verification.

In an autoregressive integrated moving average model (ARIMA), the future value of a variable is assumed to be a linear function of several past observations and random errors. The linear function is based on three parametric linear components: autoregressive (AR), integration (I), and moving average (MA) method (Box and Jenkins, 1976). Box and Jenkins (1976) popularized ARIMA models as

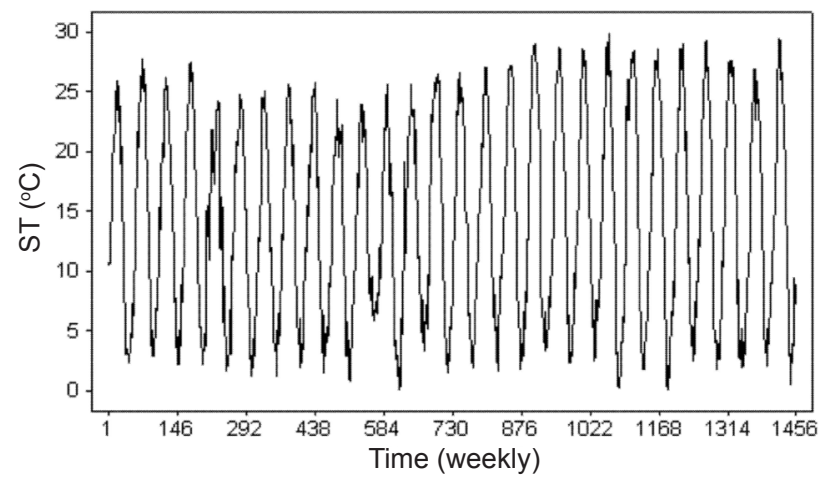

Fig. 1. Time series plot of weekly soil temperature at $10 \mathrm{~cm}$ depth for the whole period (1 January 1986 to 31 December 2013) at Bajgah Agricultural Experimental Station, Shiraz, Iran.

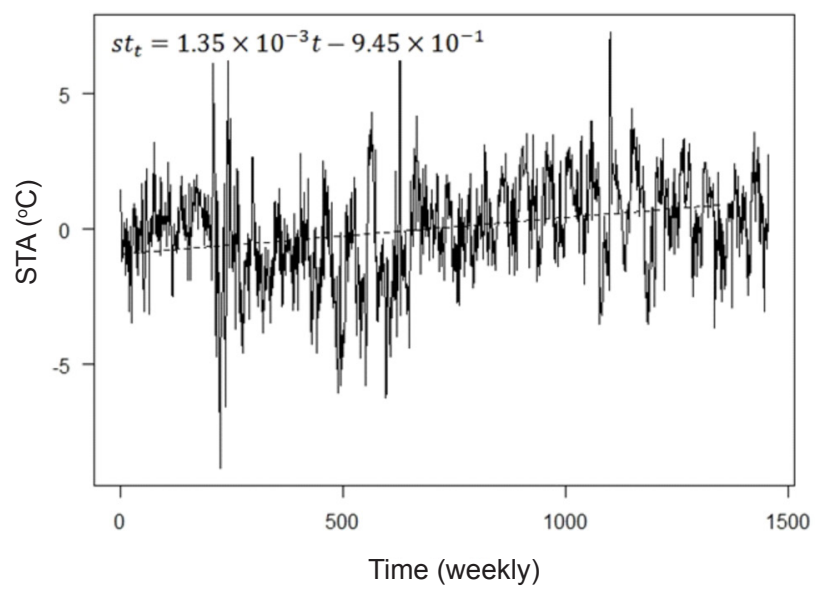

Fig. 2. Anomalies time series plot of the series in Fig. 1 and trend line for the period of 1986-2011. 
$(p, d, q)$, where $p$ and $q$ are the orders for non-seasonal $\mathrm{AR}$ and MA, respectively. The non-seasonal differencing parameter that shows how many differencing steps were used for making the series stationary is shown by $d$. The general format of the ARIMA model is:

$$
\phi_{p}(B) \nabla^{d} x_{t}=\theta_{q}(B) w_{t},
$$

where: $x_{t}$ is a time series and $w_{t}$ is the white noise random variable. The ordinary autoregressive and moving average components are, respectively, represented by:

$$
\phi_{p}(B)=1-\phi_{1} B-\phi_{2} B^{2}-\ldots+\phi_{p} B^{p},
$$

and

$$
\theta_{q}(B)=1+\theta_{1} B+\theta_{2} B^{2}+\ldots+\theta_{q} B^{q} .
$$

The ordinary difference component is defined by $\nabla^{\mathrm{d}}=(1-B)^{d}$, where $B$ is the backshift operator defined by $B^{d} x_{t}=x_{t-d}$.

The ARIMA modelling approach involves three iterative steps of model identification, parameter estimation and diagnostic checking. Identification of the general form of a model includes two stages:

- if it is necessary, appropriate differencing of the series is performed to satisfy the stationary and normality assumptions;

- the temporal correlation structure of the transformed data is identified by examining its autocorrelation function (ACF) and partial autocorrelation function (partial ACF) (Mishra and Desai, 2005).

The ACF is a useful statistical tool that determines whether earlier values in the series have some relation to later values. Partial ACF is the amount of correlation between a variable and a lag of itself that is not explained by correlations at all low order lags. Box and Jenkins (1976) proposed to use the ACF and partial ACF of sample data as the basic tools to identify the order of the ARIMA model. Considering the ACF and partial ACF graphs of soil temperature series, different ARIMA models are identified for model selection. The model that gives the minimum Akaike Information Criterion, AIC (Eq. (4)), and follows the assumptions of residual independence is selected as the best model. Shapiro-Wilk (1965) and Ljung-Box (1978) test were used to check the normality and independence of residuals, respectively. The $A I C$ equation is defined as:

$$
A I C=n(\ln ((2 \pi R S S) / n)+1)+2 m,
$$

where: $m=(p+q)$ is the number of terms estimated in the model (sum of autoregressive and moving average orders), $n$ is the number of sample size and RSS denotes the sum of squared residuals (Ömer Faruk, 2010). In the identification step, data transformation is often required to make the time series stationary. Stationarity is a necessary condition in building an ARIMA model used for forecasting. A stationary time series is characterized by constant mean and autocorrelation structure over time. When the observed time series presents a trend and heteroscedasticity, differencing and power transformation are applied to the data to remove the trend and to stabilize the variance before fitting an ARIMA model. Once a tentative model is identified, estimation of the model parameters is straightforward (Khashei and Bijari, 2011).

After the functions of the ARIMA model were specified, the parameters of the functions must be estimated. Once an appropriate model is chosen and its parameters are estimated, the methodology (Box and Jenkins, 1976) requires examining the residuals of the model to verify that the model is adequate for the series. Several tests are employed for the diagnostic check to determine whether the residuals of the selected ARIMA models from the ACF and partial ACF graphs are independent, homoscedastic and normally distributed. If the homoscedasticity and normality assumptions are not satisfied, the observations are transformed by a Box-Cox (1964) transformation (Wei, 1994). For a good forecasting model, the residuals left over after fitting the model must satisfy the requirements of a white noise process. In order to determine whether soil temperature time series are independent, the residual autocorrelation function (RACF) of the series was studied. There are several useful tests related to RACF for the independence of residuals. The first one is the correlograms drawn by plotting the residual ACF against lag number. If the ARIMA model is correct, the estimated autocorrelations of the residuals are uncorrelated and distributed approximately normally about zero. The second one is the Ljung-Box-Pierce (Box and Pierce, 1970; Ljung and Box, 1978) statistics. In order to test the null hypothesis (that a current set of autocorrelations is white noise), test statistics were calculated for different total numbers of successive lagged autocorrelations using the Box-Pierce statistics $(\mathrm{Q}(\mathrm{r})$ test $))$ to test the adequacy of the model. $\mathrm{Q}(\mathrm{r})$ values were compared to a critical test value of $\chi^{2}$ distribution with respect to their degrees of freedom at the probability level of 5\% (El-Din and Smith, 2002).

To verify the forecasting capability of the developed model, the weekly soil temperature anomalies were predicted from the first week of January 2012 to December 2013 as an independent test. For instance, when the lag time is one week, the soil temperature anomaly for the first week of January 2013 was firstly predicted, that for the second week of January 2012 was then predicted when the lag time is two weeks. After this, a new ARIMA model with the period of January 1986 to the first week of January 2012 was constructed to predict:

- the soil temperature anomaly for the second week of January 2012, when the lag time is one week, and

- the soil temperature anomaly for the third week of January 2012, when the lag time is two weeks. 
This procedure was sequentially repeated 104 times to predict the soil temperature anomalies from the first week of January 2012 to the last week of December 2013 for different lag times. Then, the observed and predicted data with the upper and lower bounds of the $95 \%$ confidence interval of the predicted values were plotted.

\section{RESULTS AND DISCUSSION}

The weekly time series plot of the soil temperature anomalies for the period from January 1986 to December 2011 which was used for constructing ARIMA models (Fig. 2). A simple linear regression analysis was applied over this time series to test the linear trend as follows:

$$
s t_{t}=\beta_{1} t+\beta_{0}+w_{t} t=1,2, \ldots, 1352,
$$

where $s t_{t}$ is the observed soil temperature, $w_{t}$ is the white noise random variable, and $t$ is the time location, starting from the first week of January 1986. The estimated slope $\beta_{1}$ was $0.00135^{\circ} \mathrm{C} /$ week, with standard error of $0.000126^{\circ} \mathrm{C} /$ week, resulting in t-value of $0.00135 / 0.000126=10.7$ which is significantly greater than the quantile of t-distribution with $\alpha=0.01$ and 1351 degree of freedom $\left(t_{0.005}(1351)=\right.$ 2.32)). The soil temperature at $10 \mathrm{~cm}$ depth has, therefore, increased by about $1.8^{\circ} \mathrm{C}$ for the period of 1986-2011. The estimated linear trend is also shown in Fig. 2.

The autocorrelation function of the surface soil temperature anomalies time series for the period of 1986-2011 shows Fig. 3. The results indicate that the autocorrelation coefficient slowly decreases as lag time increases, and the first fifteen autocorrelation coefficients are statistically significant $(p<0.05)$. Both the linear trend and the ACF indicate that there is a significant warming trend in the surface soil temperature anomalies time series of the studied area. Based on the obtained upward significant trend in soil temperature anomalies, it is expected that this parameter of soil will be increasing in the future in this region. The probable warming will lead to more evapotranspiration and to an intensification of the world water shortage crisis.

Increases in soil temperatures have been directly observed from long term measurements or inferred from borehole temperature profiles in high latitude or altitude regions of North America (Quinton et al., 2011), Asia (Wu et al., 2012) and Europe (Hipp et al., 2012).

Due to this significant positive trend, a first order differencing procedure $(d=1)$ was performed to de-trend the weekly time series data. The differenced time series plot of surface soil temperature and its fitted linear regression indicate that there is no linear trend in differenced time series data (Fig. 4). The ACF analysis of the differenced series showed significant correlations at lags 1 and 2 (Fig. 5a), suggesting that the moving average model has two or less parameters (ie $q \leq 2$ ). Since the plot of the partial autocorrelation function exhibited significant correlations for the lags fewer than 8, an AR model of order 8 was suggested

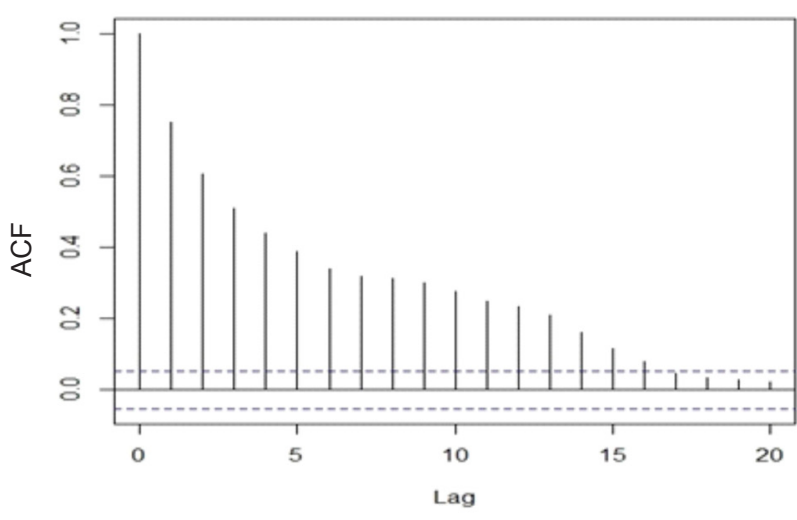

Fig. 3. Sample autocorrelation function (ACF) of the time series (the dashed lines indicate the interval of $\pm \frac{196}{\sqrt{n}}$ ).

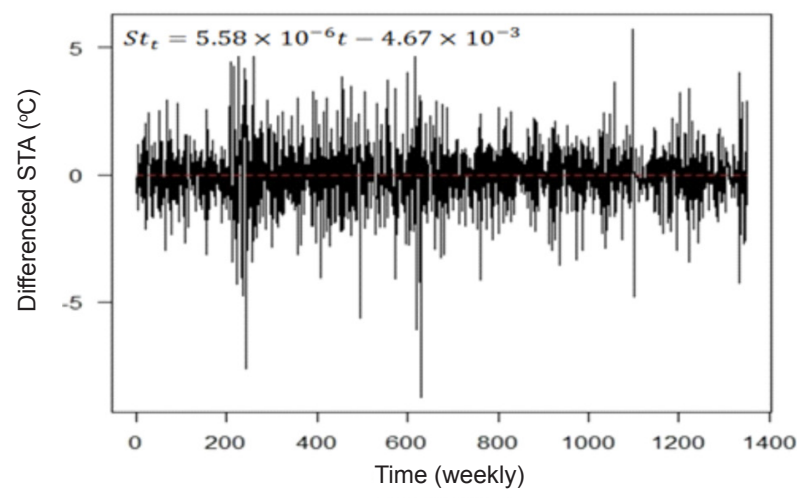

Fig. 4. Time series and trend line plots of the differenced weekly surface soil temperature anomalies time series for the period of 1986-2011.

a
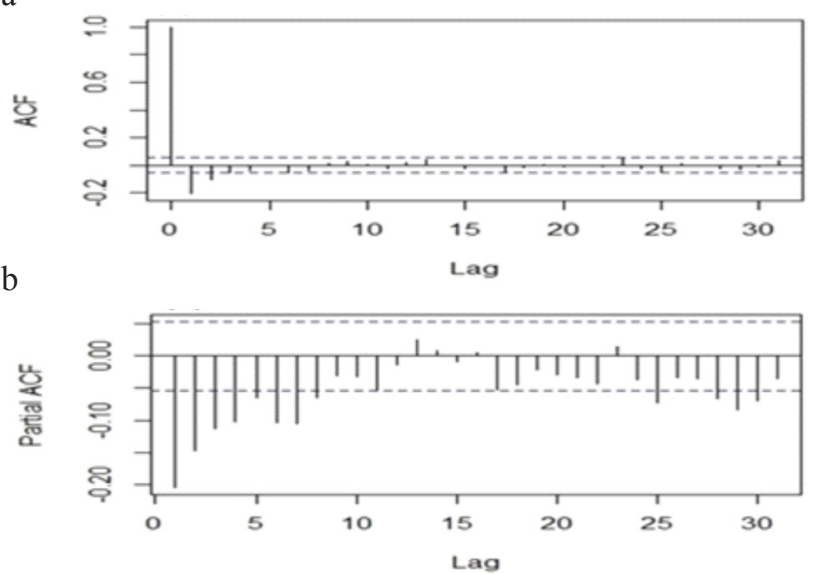

Fig. 5. (a) Sample autocorrelation function (ACF) and (b) sample partial autocorrelation function (partial ACF) of the series (the dashed lines indicate the interval of $\pm \frac{196}{\sqrt{n}}$ ). 
(Fig. 5b). After order identification, Eqs (2) and (3) were used to estimate the parameter values. The significance values of these parameters were eventually examined using the t-test. Accordingly, Table 1 indicates the characteristics of the candidate ARIMA models to predict soil temperature anomalies.

Among the candidate models, the following ARIMA $(2,1,1)$ model with the minimum value of AIC (4410.9) and the minimum value of error variance $\left(\hat{\sigma}_{w}^{2}=1.52\right)$ was the most appropriate model for the prediction of surface soil temperature.

$\left(1-0.658_{0.0274} B-0.0679_{0.0274} B^{2}\right) \nabla^{1} s t_{t}=\left(1-0.989_{0.0038} B\right) w_{t}$.

The values given in the parentheses are the standard errors of estimated parameters. All of the presented coefficients were statistically significant. For example, the t-value of $0.0679 / 0.0274=2.47$ is significantly greater than the quantile of t-distribution with $\alpha=0.01$ and 1348 degree of freedom $\left.\left(\mathrm{t}_{0.005}(1384)=2.32\right)\right)$.

To the best of our knowledge, no research has been carried out on applying the ARIMA models for the prediction of soil temperature. But, a series of studies have been done by Raghuwanshi and Wallender (1996, 1997, 1998, 1999) to develop a seasonal irrigation model. The aforementioned investigators built up an autoregressive moving average ARIMA model of $(1,0,1)$ order by applying the ACF and partial ACF of the standardized grass reference crop evapotranspiration $\left(\mathrm{ET}_{0}\right)$. They used the same model to predict both irrigation schedules and optimum furrow irrigation designs (inflow rate and cut-off time) and found that predictions could be properly explained by the underlying stochastic process. Aljoumani et al. (2012) also developed an ARIMA model of $(2,1,0)$ order to predict the soil water content at the depth of interest by measuring water content in a single depth in order to precisely determine the next irrigation time and its effect on soil water content at the depth of interest. Besides, Romilly (2005) also used anomaly time series to eliminate the seasonal effect in global monthly near-surface mean temperature data. The aforementioned study used time series techniques to model the properties of a global mean temperature dataset in order to develop a parsimonious forecasting model for managerial decision-making over the short term horizon. A forecasting evaluation showed that the chosen model performed well and the estimation results confirm the findings of a number of previous studies.

The time series plot of the standardized residuals does not show any increasing or decreasing pattern (data not shown). The ACF of these residuals indicates no apparent departure from the model assumptions, $w_{t}$ in Eq. (6) being a white noise random variable. The Ljung-Box-Pierce test statistic (Box and Pierce, 1970; Ljung and Box 1978) was significant at time lags 1 through 25 . The significant p-value of the Kolmogorov-Smirnov normality test suggests that the residuals are normally distributed. In general, according to this information, the proposed ARIMA model has been adequately fitted.

T a b l e 1. Characteristics of the ARIMA models which were candidates to predict soil temperature anomalies

\begin{tabular}{|c|c|c|c|c|c|}
\hline Model type & Model structure & Coefficients & Standard error & AIC & $\sigma_{w}^{2}$ \\
\hline \multirow{2}{*}{$\operatorname{ARIMA}(1,1,1)$} & AR1 & 0.7040 & 0.0204 & \multirow{2}{*}{4415.0} & \multirow{2}{*}{1.53} \\
\hline & MA1 & -0.9879 & 0.0041 & & \\
\hline \multirow{3}{*}{$\operatorname{ARIMA}(2,1,1)$} & AR1 & 0.6583 & 0.0274 & \multirow{3}{*}{4410.9} & \multirow{3}{*}{1.52} \\
\hline & AR2 & 0.0679 & 0.0274 & & \\
\hline & MA1 & -0.9892 & 0.0038 & & \\
\hline \multirow{6}{*}{$\operatorname{ARIMA}(3,1,3)$} & AR1 & 0.2713 & 0.0214 & \multirow{6}{*}{4415.0} & \multirow{6}{*}{1.52} \\
\hline & AR2 & -0.6768 & 0.0148 & & \\
\hline & AR3 & 0.6931 & 0.0217 & & \\
\hline & MA1 & -0.5604 & 0.0058 & & \\
\hline & MA2 & 0.5733 & 0.0075 & & \\
\hline & MA3 & -0.9837 & 0.0085 & & \\
\hline
\end{tabular}




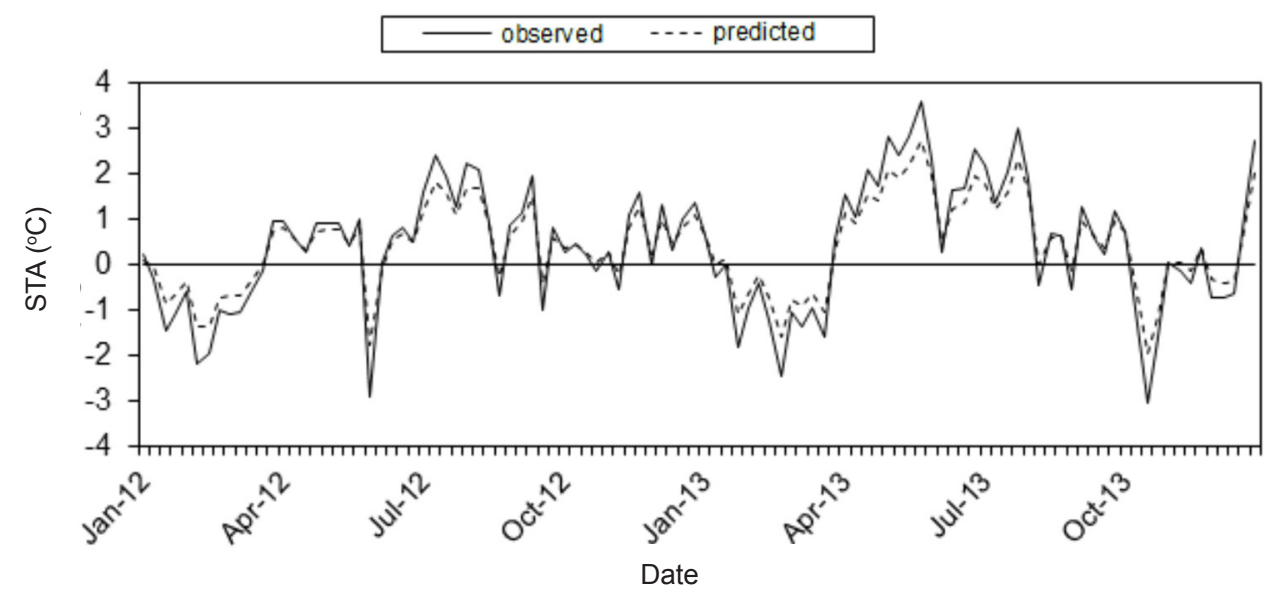

Fig. 6. Observed and predicted surface soil temperature anomalies (STA) using the proposed model, ARIMA $(2,1,1)$ for the period of January 2012 to December 2013.

The observed and predicted soil temperature anomalies for the period of January 2012 to December 2013 with lag one are shown in Fig. 6. The results indicate that the applied ARIMA model has recognized the fluctuations of soil temperature accurately. The Pearson correlation coefficient $(\mathrm{R})$ between observed and predicted soil temperature anomalies for lead times up to 3 weeks were, respectively, $0.99,0.65$ and 0.53 , all statistically significant at $1 \%$ significance level. The corresponding root mean square errors were $0.39,1.03$ and $1.18^{\circ} \mathrm{C}$. To the best of our knowledge, no research has been done on applying the ARIMA model for predicting soil temperature, but other studies have reported that ARIMA models are highly efficient in short-term forecasting different soil parameters such as soil salt and water content (Aljoumani et al., 2012; Zou et al., 2010), soil heat flux (Kumar et al., 2009) and soil dryness index (Li et al., 2003).

\section{CONCLUSIONS}

1. An autoregressive integrated moving average (ARIMA) model, characterized as ARIMA $(2,1,1)$ was developed to predict weekly values of the surface soil temperature anomalies. This developed model tends to accurately predict soil temperature anomalies.

2. The soil temperature anomalies time series were non-stationary.

3. The anomalies time series were differenced to conduct analysis in frequency domain to obtain the parameters of the ARMA models for the purposes of using the ARIMA model to predict surface soil temperature.

4. There is a high correlation coefficient between the observed and predicted surface soil temperature anomalies; that was 0.99 for lead time 1 week.

5. The indicated significant warming trend $\left(1.8^{\circ} \mathrm{C}\right.$ for the period 1986-2011) in soil temperature could improve our understanding of warming and its biological and biochemical consequences.

\section{REFERENCES}

Aljoumani B., Sànchez-Espigares J.A., Cañameras N., Josa R., and Monserrat J., 2012. Time series outlier and intervention analysis: Irrigation management influences on soil water content in silty loam soil. Agr. Water. Manag., 111, 105-114.

Box G.E. and Cox D.R., 1964. An analysis of transformations. J. R. Stat. Soc. Series B (Methodological), 211-252.

Box G.E. and Jenkins G.M., 1976. Time series analysis: forecasting and control. Holden-Day, San Francisco, CA, USA.

Box G.E. and Pierce D.A., 1970. Distribution of residual autocorrelations in autoregressive-integrated moving average time series models. J. Am. Stat. Assoc., 65(332), 1509-1526.

Chatfield C., 2013. The analysis of time series: an introduction. CRC Press, Boca Raton, FL, USA.

Diaz-Robles L.A., Ortega J.C., Fu J.S., Reed G.D., Chow J.C., Watson J.G., and Moncada-Herrera, J.A., 2008. A hybrid ARIMA and artificial neural networks model to forecast particulate matter in urban areas: the case of Temuco, Chile. Atmos. Environ., 42(35), 8331-8340.

Dorado J., Sousa E., Calha I., Gonzalez-Andujar J., and Fernandez-Quintanilla C., 2009. Predicting weed emergence in maize crops under two contrasting climatic conditions. Weed. Res., 49(3), 251-260.

El-Din A.G. and Smith D.W., 2002. A combined transfer-function noise model to predict the dynamic behavior of a fullscale primary sedimentation tank. Water. Res., 36(15), 3747-3764.

Flerchinger G. and Hardegree S., 2004. Modelling near-surface soil temperature and moisture for germination response predictions of post-wildfire seedbeds. J. Arid. Environ., $59(2), 369-385$.

Hardegree S., Flerchinger G., and Van Vactor S., 2003. Hydrothermal germination response and the development of probabilistic germination profiles. Ecol. Model., 167(3), 305-322.

Harvey S.J. and Forcella F., 1993. Vernal seedling emergence model for common lambsquarters (Chenopodium album). Weed. Sci., 309-316. 
Hipp T., Etzelmüller B., Farbrot H., Schuler T., and Westermann S., 2012. Modelling borehole temperatures in Southern Norway-insights into permafrost dynamics during the 20th and 21st century. The Cryosphere, 6(3), 553-571.

Houle D., Duchesne L., Ouimet R., Paquin R., Meng F.-R., and Arp P.A., 2002. Evaluation of the FORHYM2 model for prediction of hydrologic fluxes and soil temperature at the Lake Clair Watershed (Duchesnay, Quebec). Forest. Ecol. Manag., 159(3), 249-260.

Kärner O., 2009. ARIMA representation for daily solar irradiance and surface air temperature time series. J. Atmospheric Solar-Terrestrial Physics, 71(8), 841-847.

Khashei M. and Bijari M., 2011. A novel hybridization of artificial neural networks and ARIMA models for time series forecasting. Appl. Soft. Comput., 11(2), 2664-2675.

Kumar M., Kumar A., Mahanti N., Mallik C., and Shukla R.K., 2009. Surface flux modelling using ARIMA technique in humid subtropical monsoon area. J. Atmospheric Solar-Terrestrial Physics, 71(12), 1293-1298.

Li Y., Campbell E., Haswell D., Sneeuwjagt R., and Venables W., 2003. Statistical forecasting of soil dryness index in the southwest of Western Australia. Forest. Ecol. Manag., 183(1), 147-157.

Ljung G.M. and Box G.E., 1978. On a measure of lack of fit in time series models. Biometrika, 65(2), 297-303.

McKenzie E., 1984. General exponential smoothing and the equivalent ARMA process. J. Forecasting, 3(3), 333-344.

Mishra A. and Desai V., 2005. Drought forecasting using stochastic models. Stoch. Env. Res. Risk. A., 19(5), 326-339.

Monin A., Yaglom A., and Lundgren T., 1976. Statistical fluid mechanics. J. Appl. Mech., 2(43), 521.

Ömer Faruk D., 2010. A hybrid neural network and ARIMA model for water quality time series prediction. Eng. Appl. Artif. Intel., 23(4), 586-594.

Pektaş A.O. and Kerem Cigizoglu H., 2013. ANN hybrid model versus ARIMA and ARIMAX models of runoff coefficient. J. Hydrol., 500, 21-36.

Persson T. and Wirén A., 1995. Nitrogen mineralization and potential nitrification at different depths in acid forest soils. Plant Soil, 168(1), 55-65.

Quinton W., Hayashi M., and Chasmer L., 2011. Permafrostthaw-induced land-cover change in the Canadian subarctic: implications for water resources. Hydrol. Process, 25(1), $152-158$

Raghuwanshi N. and Wallender W., 1996. Modeling seasonal furrow irrigation. J. Irrig. Drain. E-ASCE, 122(4), 235-242.
Raghuwanshi N. and Wallender W., 1997. Field-measured evapotranspiration as a stochastic process. Agric. Water. Manag., 32(2), 111-129.

Raghuwanshi N. and Wallender W., 1998. Optimization of furrow irrigation schedules, designs and net return to water. Agric. Water. Manag., 35(3), 209-226.

Raghuwanshi N. and Wallender W., 1999. Forecasting and optimizing furrow irrigation management decision variables. Irrigation Sci., 19(1), 1-6.

Romilly P., 2005. Time series modelling of global mean temperature for managerial decision-making. J. Environ. Manag., 76(1), 61-70.

Roundy B.A. and Call C., 1988. Revegetation of arid and semiarid rangelands In: Vegetation Science Applications for Rangeland Analysis and Management (Ed. P.T. Tueller). Springer, Netherlands.

Schütt M., Borken W., Spott O., Stange C.F., and Matzner E., 2014. Temperature sensitivity of $\mathrm{C}$ and $\mathrm{N}$ mineralization in temperate forest soils at low temperatures. Soil. Biol. Biochem., 69, 320-327.

Shahwan T. and Odening M., 2007. Forecasting agricultural commodity prices using hybrid neural networks In: Computational Intelligence in Economics and Finance (Eds S.H. Chen, P.P. Wang, T.W. Kuo). Springer Press, Berlin Heidelberg.

Shapiro S.S. and Wilk M.B., 1965. An analysis of variance test for normality (complete samples). Biometrika, 591-611.

Subke J.-A. and Bahn M., 2010. On the 'temperature sensitivity' of soil respiration: Can we use the immeasurable to predict the unknown? Soil. Biol. Biochem., 42(9), 1653-1656.

Toosi E.R., Schmidt J.P., and Castellano M.J., 2014. Soil temperature is an important regulatory control on dissolved organic carbon supply and uptake of soil solution nitrate. Eur. J. Soil. Biol., 61, 68-71.

Wei W.W.-S., 1994. Time series analysis. Addison-Wesley Press. Wu Q., Zhang T., and Liu Y., 2012. Thermal state of the active layer and permafrost along the Qinghai-Xizang (Tibet) Railway from 2006 to 2010. The Cryosphere, 6(3), 607-612.

Zhang G.P., 2003. Time series forecasting using a hybrid ARIMA and neural network model. Neurocomputing, 50, 159-175.

Zou P., Yang J., Fu J., Liu G., and Li D., 2010. Artificial neural network and time series models for predicting soil salt and water content. Agric. Water Manag., 97(12) 2009-2019. 